\title{
Prevalence of Impaired Glucose Tolerance and its Utility to Predict Prognosis of Patients with Liver Cirrhosis
}

\author{
praveen bugalia
}

\section{Abstract:}

Background and Aims: Cirrhosis of liver is one of the commonest cause of ill health among general population particularly in chronic alcoholics in developing countries like India. Cirrhosis patients show increased incidence of hyperglycemia; to study impaired glucose tolerance in patient with cirrhosis \& to predict prognosis of patients with Liver Cirrhosis.

50 Patients of liver cirrhosis of either sex and age were included in the study. All patient were subjected to oral glucose tolerance test and the findings were correlated with the severity of the disease.

Results: $16 \%$ patients belonged to child's grade A, 50\% belonged to grade B \& $34 \%$ patients belonged to Child's grade C. $56 \%$ of patients showed impaired glucose tolerance. No patient showed diabetic response to glucose tolerance test. $15(88 \%)$ patients belonging to Child's grade $\mathrm{C}$ showed impaired glucose tolerance. While there were 12 (48\%) patients in Child's grade B who showed impaired glucose tolerance. Only $1(12.5 \%)$ patient in Child's grade A had glucose intolerance.

Conclusion: The incidence of impaired glucose tolerance was significantly high among patients with cirrhosis of liver. This high incidence of glucose intolerance was not related to the age, sex and etiology but was related with the severity of disease.

Cirrhosis of liver is one of the commonest cause of ill health among general population particularly in chronic alcoholics in developing countries like India. Since it is a slowly progressive silent disease it is usually overlooked by the patient themselves unless they develop some complication. In India there has been a rise in case of post necrotic cirrhosis following hepatitis B infection.

Cirrhosis a generic term, includes all forms of chronic diffuse liver disease characterized by significant loss of liver cells, collapse and diffuse septal fibrosis of liver associated with regenerative parenchymal nodules and disturbed intrahepatic circulation. Cirrhosis results from prolonged and widespread but patchy hepatocellular necrosis which may have many cause (Oxford Textbook of Medicine $3^{\text {rd }}$ edition, pg. 2085). ${ }^{1}$

Cirrhosis of liver causes significant derangement of carbohydrate metabolism. Narayan (1906) first time, established impairment of carbohydrate metabolism scientifically in liver disease including cirrhosis of liver. Impaired glucose tolerance is related to severity of liver disease and there is no evidence that DM predispose to cirrhosis. 0 Riggio et al. (1982). ${ }^{2}$ Glucose intolerance in cirrhosis is caused by simultaneous occurrence of insulin resistance in muscles and inadequate secretion of insulin by beta-cell of pancreas to overcome the insulin resistance (Petrides et al, 1994). ${ }^{3}$

Cirrhosis patients show increased incidence of hyperglycemia associated with increased insulin level. Hyperglycemia is explained by reduced absorption of glucose by muscles and decreased glycogen storage in liver and muscles. This leads to increased insulin level in serum and increased insulin resistance [Sleisenger \& Fordtrans GI and liver ds. $6^{\text {th }}$ Edi. Vol 2. Page 1067]. ${ }^{4}$ 
Fasting blood glucose is usually normal in cirrhotics but most are insulin resistant and are intolerant to an oral glucose load, and have post prandial hyperglycemia (Oxford Textbook of Medicine, $3^{\text {rd }}$ edition, Pg. 2088). ${ }^{1}$

In this study oral glucose tolerance test was done in patients of cirrhosis to demonstrate glucose intolerance and its correlation with the severity of cirrhosis.

\section{MATERIALS AND METHODS}

The study was conducted on 50 patient with cirrhosis of liver, irrespective of etiology who were admitted in medicine ward, department of medicine, Dr. BSA Hospital, Delhi during study period. All the cases of liver cirrhosis of either sex and age were included in the study.

All patient were subjected to oral glucose tolerance test and the findings were correlated with the severity of the disease.

Detailed history was taken and patients were subjected to thorough clinical examination and necessary investigations.

\section{Inclusion Criteria}

Patient's diagnosed clinically, biochemically and ultrasonographically as having cirrhosis.

\section{Exclusion Criteria}

1) History of diabetes mellitus / family history of diabetes mellitus

2) Other endocrine disorders affecting carbohydrate metabolism

3) Patient on drugs which alter glucose metabolism

Oral Glucose Tolerance Test

$75 \mathrm{gm}$ of anhydrous glucose in $250-300 \mathrm{ml}$ of water was given orally to patients. Blood samples were collected before (fasting) \& $30 \mathrm{~min}, 60 \mathrm{~min}, 120 \mathrm{~min}$, after the glucose load.

\section{RESULTS}

This study involved 50 adult patients with liver cirrhosis in whom oral glucose tolerance test was performed. Patients were classified into Child's grade A, B and C based on the level of Jaundice, serum albumin, ascites, encephalopathy and prothrombin time.

The highest incidence of liver cirrhosis was among patients between the ages of 41-50 years (38\%), lowest incidence was found among younger age group (11-20 years - 2\%). Mean age was found to be $45 \pm 4$ years.

Alcoholism was found as the causative factor in $34 \%$ of patients and hepatitis B virus in $26 \%$ cases. In $40 \%$ etiology could not be ascertained. Probably it was due to nutritional and other causes.

Serum bilirubin above $3 \mathrm{mg} / \mathrm{dL}$ was found in $36 \%$ of patients, $16 \%$ patients had serum bilirubin level between 2 and $3 \mathrm{mg} / \mathrm{dL}$ and in $48 \%$ patients it was below $2 \mathrm{mg} / \mathrm{dL}$.

Serum albumin level below $3 \mathrm{gm} / \mathrm{dL}$ was found in $72 \%$ cases whereas $18 \%$ patient had levels of $>3.5 \mathrm{gm} / \mathrm{dL} \&$ $10 \%$ patients had serum albumin leves between $3-3.5 \mathrm{gm} \%$.

$58 \%$ of patients had prothrombin time prolonged by $<4$ seconds. Only $10 \%$ showed prothrombin time prolonged 
American Research Journal of Medicine and Surgery (ARJMS)

by $>6$ seconds, $32 \%$ patients had prothrombin time prolonged in the range of 4-6 seconds.

$16 \%$ patients belonged to child's grade A, 50\% belonged to grade B \& 34\% patients belonged to Child's grade C.

Table 1. Demographic and clinical characteristics of Patients

\begin{tabular}{|c|c|c|}
\hline \multicolumn{2}{|l|}{ Male } & $38(76 \%)$ \\
\hline \multicolumn{2}{|l|}{ Female } & $12(24 \%)$ \\
\hline \multicolumn{2}{|l|}{ Age (Mean) } & $45 \pm 4$ \\
\hline \multirow{3}{*}{ Cirrhosis Etiology } & Alcohol & $17(34 \%)$ \\
\hline & HBV & $13(26 \%)$ \\
\hline & Others & $20(40 \%)$ \\
\hline \multirow{3}{*}{ Serum Bilirubin } & $<2$ & $24(48 \%)$ \\
\hline & $2-3$ & $8(16 \%)$ \\
\hline & $>3$ & $18(36 \%)$ \\
\hline \multirow{3}{*}{ Serum Albumin } & $>3.5$ & 9 (18\%) \\
\hline & $3-3.5$ & $5(10 \%)$ \\
\hline & $<3$ & $36(72 \%)$ \\
\hline \multirow[b]{3}{*}{ (Sec.) } & $0-4$ & $29(58 \%)$ \\
\hline & $4-6$ & $16(32 \%)$ \\
\hline & $>6$ & $5(10 \%)$ \\
\hline \multirow{3}{*}{ Child-Pugh } & A & $8 \quad(16 \%)$ \\
\hline & B & $25(50 \%)$ \\
\hline & $\mathrm{C}$ & $17(34 \%)$ \\
\hline
\end{tabular}

$56 \%$ of patients showed impaired glucose tolerance. No patient showed diabetic response to glucose tolerance test.

Table 2. Results of Glucose Tolerance Test

\begin{tabular}{|l|c|c|c|}
\hline No. of patients & Normal glucose tolerance & $\begin{array}{c}\text { Impaired glucose toler- } \\
\text { ance }\end{array}$ & Diabetes \\
\hline & $22(44 \%)$ & $28(56 \%)$ & 0 \\
\hline
\end{tabular}

15 (88\%) patients belonging to Child's grade C showed impaired glucose tolerance. While there were 12 (48\%) patients in Child's grade B who showed impaired glucose tolerance. Only $1(12.5 \%)$ patient in Child's grade A had glucose intolerance.

Table 3. Distribution of impaired glucose tolerance in patients of child's grade

\begin{tabular}{|c|c|c|}
\hline Child's grade & No. of patients & Impaired glucose tolerance \\
\hline A & 8 & $1(12.5 \%)$ \\
\hline B & 25 & $12(48 \%)$ \\
\hline C & 17 & $15(88 \%)$ \\
\hline
\end{tabular}

Volume 2 


\section{DISCUSSION}

The liver plays a key role in the carbohydrate metabolism. It maintains the glucose homeostasis through glycogenesis, glycogenolysis, glycolysis and gluconeogenesis. In the fasting state, the liver contributes to glucose homeostasis by glycogenolysis and gluconeogenesis in response to hypo-insulinaemia and hyperglucagonemia. Since the liver has considerable reserve capacity, minimal or even moderate cell injury may not cause disturbance in the glucose homeostasis. However in advanced liver disease, derangements do occur in metabolic pathways. Abnormalities of glucose homeostasis are common in cirrhosis.

Morales et al. $(1990)^{5}$ reported that the severity of liver dysfunction correlates with alterations in carbohydrate metabolism.

\section{Incidence of Glucose Intolerance}

Impaired glucose tolerance was noticed in $56 \%$ of the patients. Among these 15 patients were from grade $\mathrm{C}$, 12 patients belonged to grade B and 1 patient belonged to grade A. The incidence of glucose intolerance was $88 \%$ in patients with advanced liver disease (Child's grade ' $C$ '). In patients who belonged to grade ' $\mathrm{B}$ ' $48 \%$ had glucose intolerance. Only $12.5 \%$ of patients showed glucose intolerance among those who were classified as Child's grade 'A'. Study by Muller et al $(1994)^{6}$ in which 108 patients with liver cirrhosis were subjected to oral glucose tolerance test, showed that $35 \%$ patients had glucose intolerance of which $63 \%$ of patients belonged to Child's grade $\mathrm{C}$.

This study shows that the incidence of glucose intolerance observed in patients with liver cirrhosis is related to the severity of liver cell injury. Higher incidence of glucose intolerance had been observed in the patients with advanced stage of disease. There was no significant relationship to the variables such as age, sex and aetiology of cirrhosis. Carbone et al. (1993) ${ }^{7}$ found that there was no relationship between the incidence of glucose intolerance and age, sex and family history of diabetes. Jacobo et al. $(1996)^{8}$ reported lack of relationship between the prevalence of glucose intolerance and aetiology of cirrhosis of liver.

Custro $\mathrm{N}$ et al, $2001^{9}$ who studied glycemic homeostasis in chronic viral hepatitis and liver cirrhosis concluded that increase in prevalence of IGT/DM occurred independent of age and sex but in relationship with severity of disease.

The underlying mechanism of glucose intolerance is complex and not fully understood (Kruszynska, 1993) ${ }^{10}$. There is peripheral insulin resistance and reduced insulin clearance by most cirrhotics (Taylor, 1985) ${ }^{11}$. Peripheral hyperinsulinism results from both a higher insulin secretion rate and a redued insulin hepatic clearance (Scheen et al., 1993) ${ }^{12}$.

Walker et al, (1993) ${ }^{13}$ reported that the glucose intolerance in cirrhosis is characterized by insulin stimulated non-oxidative glucose disposal. The high non esterified fatty acid seen in liver cirrhosis most likely do not contribute to this effect. Splanchnic glucose uptake is normal in cirrhosis.

Eiichi Imano et al (1999) ${ }^{14}$ studied impaired splanchnic and peripheral glucose uptake in liver cirrhosis. These results suggest that glucose intolerance in patients with liver cirrhosis is caused by a defect of the glucose uptake in both splanchnic and peripheral tissues.

In liver cirrhosis adipocytes show a diminished insulin sensitivity. The first pass hepatic extraction of insulin 
is reduced as compared with controls and most patients compensate for the peripheral insulin resistance with increased pancreatic insulin secretion. This results in high circulating insulin levels, normal fasting blood glucose and minimal glucose intolerance.

Approximately one half to one third of patients with liver cirrhosis show glucose intolerance after an oral glucose load. Such cirrhotic patients show a normal fasting blood glucose and a high circulating insulin level in general. The underlying mechanism of this glucose intolerance has not been fully elucidated but can be mainly explained by the insulin resistance of the patients. However, recent studies have revealed that glucose tolerance is determined not only by the insulin sensitivity of peripheral tissues and the liver but also by glucose sensitivity. The latter is defined as the ability of glucose to enhance its disappearance per se from plasma independently of the plasma insulin status. In liver cirrhosis, insulin resistance due to damaged insulin receptor molecules and impaired intracellular signal transduction via the receptors has been well described. In addition, several recent studies showed that glucose sensitivity was also reduced in liver cirrhosis.

To summarize it can be concluded from this study that the incidence of impaired glucose tolerance was significantly high among patients with cirrhosis of liver. This high incidence of glucose intolerance was not related to the age, sex and etiology but was correlated with the severity of disease and poor prognosis.

\section{REFERENCES}

1. Oxford Textbook of Medicine $3^{\text {rd }}$ edition, pg. 2085-2088.

2. O. Riggio, M. Merli, C. Cangiano et al. Glucose intolerance in liver cirrhosis. Metabolism Vol. 31, Issue 6, June 1982, 627-634.

3. Petrides AS, Groop LC, Riely CA, et al. Effect of physiologic hyperinsulinemia on glucose and lipid metabolism in cirrhosis.

4. Sleisenger \& Fordtrans en \& liver P1067,1061. $6^{\text {th }}$ edition volume 2.

5. Morales et al. Reported that the severity of liver dysfunction correlates with alterations in carbohydrate metabolism. 1990.

6. Muller MJ, Pirlich M, Balk SHJ et al. Glucose intolerance in liver cirrhosis: Role of hepatic and non hepatic influences, Eur J Clin Chem Biochem, 1994 Oct; 32 (10): 749-58.

7. Carbone L, Del vecchio Blanco C,et al, Incidence of altered glucose tolerance in liver cirrhosis. 1993 Oct-Nov;22(1):37-44.

8. Jacobo et al. Study of etiological agent in cirrhosis. 1996.

9. Custro N; Carroccio A; Ganei et al. Glycemic homeostasis in chronic viral hepatitis and liver cirrhosis. Diabetes and metabolism. Vol. 27, Issue 4, Part 1, 2001, Pages 476-481.

10. Kruszynska YT, Meyer-Alber A, Darakhshan F, et al. Metabolic handling of orally administered glucose in cirrhosis.1993 Mar;91(3):1057-66.

11. Taylor KJW, Riely CA, Hammers et al: Quantitative USG attenuation in normal liver and in patient with diffuse liver disease importance of fat Radiology 1986 160: 65: 71.

Volume 2

Page 5 
12. Scheen AJ, Gerald PL, Letiexhe MR< et al. Insulin secretion, clearance, and action on glucose metabolism in cirrhotics. 1993 Nov;77(5):1263-8.

13. E Shmueli, M Walker, G Alberti and CO Record, Normal splanchnic but impaired peripheral insulin-stimulated glucose uptake in cirrhosis. Hepatology 18 (1993), pp. 86-95.

14. Eiichi Imano, Tsutomu Kanda, Yoshihisa Nakatani et al: Journal of Hepatology Impaired splanchnic \& peripheral glucose uptake in liver cirrhosis. Vol. 31, Issue 3, 1999, Pg 469-473.

Citation: praveen bugalia, "Prevalence of Impaired Glucose Tolerance and its Utility to Predict Prognosis of Patients with Liver Cirrhosis". ARJMS Volume 2; pp:1-6

Copyright (c) 2016 praveen bugalia, This is an open access article distributed under the Creative Commons Attribution License, which permits unrestricted use, distribution, and reproduction in any medium, provided the original work is properly cited. 